Published in final edited form as:

Exp Neurol. 2011 April ; 228(2): 157-159. doi:10.1016/j.expneurol.2010.12.015.

\title{
Modeling of cerebellar hemorrhage
}

\author{
Gary A. Rosenberg \\ Department of Neurology, University of New Mexico Health Sciences Center, Albuquerque, NM \\ 87131, USA
}

Gary A. Rosenberg: grosenberg@salud.unm.edu

\section{Introduction}

Cerebellar hemorrhage is a life-threatening condition that is one of the few stroke syndromes that is benefited by surgery. C. Miller Fisher described the clinical features of cerebellar hemorrhage and the cardinal features of ataxia, cranial nerve palsies, and hemiparesis that indicated brainstem compression and signaled the need for surgery for a potentially lifethreatening situation (Fisher et al., 1965). This remarkable paper appeared before the introduction of computed tomography (CT) and magnetic resonance imaging (MRI). More recently, the use of CT and MRI has removed some of the uncertainty in the diagnosis, but the diagnosis can be difficult, particularly when a single symptom, such as vertigo, is the only warning of the mass in the cerebellum, and the treatment still is controversial. The key to successful treatment is twofold: 1) suspicion of a cerebellar bleed based on the sequence of events as revealed by the history rather than reliance on the examination alone, and 2) the correct choice of imaging modalities. Even with the most careful history, neurological examination and MRI scan with attention to the posterior fossa, selection of patients for surgery and timing of the surgery differ among neurosurgeons.

Following C. Miller Fisher's report, several large series of patients showed the treacherous course of the illness with death occurring either rapidly from early brainstem compression or after a delay due to the consequences of the mass impinging on the brainstem from below and the hydrocephalus distorting the brainstem from above (Ott et al., 1974). The typical patient with cerebellar hemorrhage is hypertensive. At the onset there is a severe headache followed shortly by difficulty walking due to the cerebellar lesion. An interval separates the initial symptoms from the second phase in which the growing mass presses on the brainstem causing sixth and seventh cranial nerve palsies. As the mass progressively enlarges and the tissue destruction causes edema, the brainstem is compressed and hemiparesis occurs. Without surgical removal of the mass, death may result in 24 to $72 \mathrm{~h}$. Occasionally the progression to death is prolonged, but the disease has a very high mortality rate without surgery.

\section{Neuroimaging in cerebellar hemorrhage}

While CT improved diagnostic accuracy, some hemorrhagic masses close the surface of the cerebellum were obscured by bone, and after the discovery of MRI, it replaced CT as the ideal diagnostic test. Once the clinical diagnosis is confirmed by MRI, patients are admitted to an intensive care unit where they are carefully watched and taken to surgery if the signs of progressive disease are seen. In spite of ability to diagnose masses in cerebellum and plan treatment, patients still die from this disease, and there is a need for additional treatments. Progress in finding medical treatments to use alone or in conjunction with surgery has been slower than with supratentorial masses due to the lack of animal models. 


\section{Animal models in intracerebral hemorrhage}

Several animal models have been used to study the effect of different drugs and surgical intervention on supratentorial intracerebral hemorrhage (ICH). Such studies have focused on strategies to reduce the damage from tissue compression by the mass lesion and the toxicity of the blood products (Xi et al., 2006). Two models are generally accepted in studies of ICH (Rosenberg et al., 2008). Direct injection of autologous blood models the mass effect and the toxicity of the blood products, while direct injection of bacterial collagenase provides an alternative model that more closely resembles the natural history of ICH since the mass enlarges over time and is well localized (Rosenberg et al., 1990).

Lekic et al. (2011) have extended the collagenase-induced hemorrhage model to the cerebellum and have developed a model of cerebellar hemorrhage that takes advantage of the extensive studies done with bacterial collagenase in basal ganglia lesions.. They used two doses bacterial collagenase in the studies. The lower dose of 0.2 units, which was similar to that used in supratentorial lesions, lead to a hemorrhagic mass that was too small to produce major symptoms. However, the larger dose of 0.6 units proved to be optimal. Using this model with the higher dose of collagenase, they performed a wide variety of analyses and have carefully characterized the model. This method, according to the authors, is an improvement over the injection of blood into the cerebellum because the bleeding is contained in the region of injection rather than spreading into other sites. In addition it provides an expanding lesion, which more closely mimics the human condition.

\section{Bacterial collagenase-induced hemorrhage}

Bacterial collagenase produces a dose-dependent hemorrhagic mass when directly injected into the brain. Most studies were done with injection into the basal ganglia. Collagenase attacks the type IV collagen in the basal lamina around the cerebral blood vessels, causing bleeding. Histological studies show multiple "ball" hemorrhages around blood vessels shortly after collagenase injection. With time the multiple sites form into a mass lesion (Rosenberg et al., 1990). Gradual resolution of the mass lesion with absorption and cyst formation takes place over three to four weeks. Behavioral studies show a concomitant improvement over several weeks as the blood is resorbed.

After several hours there is production of endogenous matrix metalloproteinases (MMPs) (Rosenberg et al., 1994). Continued damage is caused by the MMPs, which amplify the injury initiated by the bacterial product. The primary MMPs involved in the secondary damage are the gelatinases, 72-kDa type IV collagenase (MMP-2) and 92-kDa type IV collagenase (MMP-9). Less well studied, but important in inflammatory protease production is stromelysin-1 (MMP-3), which is a major product of macrophage/microglia activation (Gurney et al., 2006). Both brain cells and invading white blood cells are the sources of the MMPs. Neutrophils contain MMP-9 in an active form that is packaged in packets released at the site of inflammation, but the contribution of MMP-9 from neutrophils and that from brain cells is controversial (Maier et al., 2004). Other white blood cells and brain cells induce MMPs through activation of inflammatory cascades.

Matrix metalloproteinases are present in a latent form in the brain and require activation for them to act on the extracellular matrix. MMP-2 is a constitutive enzyme that is present in large quantities normally in the brain and can be measured normally in the cerebrospinal fluid. Under hypoxic and inflammatory conditions, activation of MMP-2 is achieved by a membrane-type metalloproteinase (MT-MMP-14), which is, in turn, activated by the convertase, furin. In human supratentorial bleeding very high levels of MMP-9 are detected in the blood (Montaner et al., 2001). The levels of MMP-9 correlate with the extent of intracranial bleeding. Similar studies have not been done in cerebellar hemorrhage. 


\section{Bacterial collagenase-induced cerebellar hemorrhage}

Lekic et al. (2011) have adapted the collagenase-induced hemorrhage model to the cerebellum, and have carefully defined the consequences of the mass lesion on a number of variables. Rats with cerebellar lesions produced by 0.6 units had increased brain water, elevated levels of MMP-2 and MMP-9, including the active forms of both enzymes, and reduced levels of the type IV collagen. Behavioral testing showed a pattern highly consistent with the human disease with ataxic symptoms gradually resolving over several weeks. The reason that 0.6 units of bacterial collagenase was required to produce a significant mass lesion in the cerebellum, while 0.2 was sufficient for the supratentorial lesions, is unclear. In the basal ganglia-injected animals a series of small hemorrhages are seen, which suggests that multiple sites of bleeding are occurring within a short time. Possibly the vasculature of the cerebellum is less dense or less sensitive. It is also possible that the higher amount of white matter in the cerebellum than found in the basal ganglia has an influence. Bleeding in the white matter of the cerebellum may be less intense than in the more vascular gray matter of the basal ganglia. Future studies of the early stages of the hemorrhage formation will be needed to resolve this question. However, once the mass is established with the higher dose of collagenase, it produced findings similar to those seen in the basal ganglia lesions. Edema was present at $24 \mathrm{~h}$, and both the injected and contralateral cerebellar hemispheres showed the increase in water content.

MMP measurements showed an increase in the active forms of MMP-2 and MMP-9. The injury to the blood vessels was well documented by the fall in both type IV collagen and zona ocludens-1 (ZO-1). The loss of type IV collagen indicates that the basal lamina is disrupted, while ZO-1, which is inside the endothelial cells, indicates injury to the capillaries. Tight junction proteins were not measured and it would be interesting to see if they are reduced since loss of proteins that form the tight junctions in the cerebral capillaries is characteristic of the MMP-mediated injury (Yang et al., 2007).

An important aspect of the report by Lekic and colleagues is the extensive behavioral testing that they used. The battery of behavioral tests included those for motor and cerebellar function as well as multiple cognitive tests. Motor testing showed marked ataxia with gradual recovery over 30 days. Tests of balance and locomotion showed the same pattern, but hind limb function remained impaired after 30 days. An unexpected finding of the study is the persistent cognitive deficits seen on water maze testing. This suggests that the cerebellum in the rat has a role not only in the motor system, but also in cognition, which is consistent with the data in humans with cerebellar lesions, suggesting that collagenaseinduced cerebellar hemorrhage may be a useful model for the study of the impact of cerebellar lesions on cognition in rats (Strick et al., 2009).

Collagenase caused a large cystic lesion in the cerebellum that had a profound effect on the granular layer. The circumscribed cystic lesions seen with collagenase are similar to that produced in the human after resolution of the bleeding. We will have to await further reports with additional histology to fully understand the consequences at the cellular level.

\section{Comparison of rat cerebellar hemorrhage with the disease in humans}

While this is a unique model for cerebellar hemorrhage in the rat that has many features of the disease seen in humans, there are aspects that will limit its utility. One of the main problems with using rat models to study human cerebellar hemorrhage is the basic structure of the rat brain. Rats have an elongated central nervous system with the hippocampus above the brain close to the top of the skull and a cerebellum that stretches out caudal to the supratentorial contents. During evolution, the human brain has folded into a more angular shape. The hippocampus has descended with the temporal lobes beneath the hemispheres 
and the cerebellum has taken a position lateral to the brainstem. The horizontal placement of the cerebellum in the rat limits compression of brainstem structures with expansion of the mass lesion, which is one of the cardinal features of cerebellar hemorrhage in human, and the one that causes compression of the fourth ventricle with life-threatening hydrocephalus. Brainstem compression is generally the cause of the progressive neurological deterioration in patients with large cerebellar masses, and it is the reason that surgical evacuation of the mass generally produces such satisfying results for the surgeon and the patient. In the collagenase-induced cerebellar hemorrhage model none of the animals developed hydrocephalus. This could be due to the smaller size of the lesion or the intrinsic structure of the rat brain. Probably with larger lesions from higher doses of collagenase there would be compression of the brainstem with hydrocephalus. On the positive side, the present dose of collagenase produces a significant lesion without causing death of the animal, thus allowing for the study of long-term consequences of bleeding on cerebellar tissues.

\section{Treatment of cerebellar hemorrhage}

Intracerebral hemorrhage has generated considerable controversy over the past 150 years. Most of that debate has centered on the mechanisms of injury of the supratentorial lesions with very little discussion in the literature about the pathophysiology of bleeding into the cerebellum. Two schools of thought have emerged over the past century. On the one hand, there are advocates for the rupture of a vessel, generally one that has been damaged by hypertension, with a rapid collection of blood and a marked mass effect. CharcotBourchard aneurysms described initially by Charcot's student Bourchard, are seen in the basal ganglia and are postulated to be the consequence of the change in pressure between the large middle cerebral arteries and the smaller penetrating lenticulostriate arteries. Another school of thought has proposed that the hemorrhagic mass is the result of an ischemic injury with the release of proteolytic enzymes that attack the blood vessels and lead to hemorrhage (Zulch and Minckler, 1971). The model developed by Lekic and colleagues should help shed light on this area of understudied pathophysiology. This hemorrhagic transformation is generally seen as a later consequence of an ischemic infarction, but on occasion can occur rapidly. Once the mass is formed and compresses the surrounding tissue, the mechanism of its origin becomes less important.

Little is understood about the growth of the mass lesion after an intracerebral bleed. Several studies have shown an increase in size that occurs over 12 to 24h (Broderick et al., 2007). The impact of the hemorrhage on the surrounding tissue has been extensively studied in the supratentorial bleeds, but not in the cerebellar hemorrhages. The supratentorial mass does not appear to exert sufficient pressure on the surrounding tissues to restrict cerebral blood flow,(Powers et al., 2001) but the toxic molecules released by the breakdown of blood products, particularly the thrombin, seem to have a deleterious effect (Hua et al., 2009). All of these studies have been done in tissues other than the cerebellum and with this new model it should be possible to determine if the cerebellum responds differently to bleeding.

Treatment of cerebellar hemorrhage in humans remains controversial in spite of the passage of over 50 years since the reports on surgical treatment. Large masses with signs of brainstem compression require urgent ventriculostomy and surgical removal of the mass. When the patient has a mass less than $3 \mathrm{~cm}$ and is stable, the treat of deterioration is difficult to judge. Some neurosurgeons favor removal of the mass before the patient deteriorates, while others prefer to watch the patient carefully and intervene when deterioration begins. With the ability to visualize the mass and the hydrocephalus with CT, the watchful waiting approach is generally favored. Some neurosurgeons place a catheter in the ventricle to prevent the consequences of hydrocephalus. 
Collagenase-induced cerebellar hemorrhage will provide useful information on the effect of bleeding on the cerebellar tissues, and the mechanisms of healing. Drugs that block the inflammatory response around the hematoma have been tested in models of intracerebral hemorrhage. Minocycline, a tetracycline derivative with anti-inflammatory effects that blocks the action of MMPs, reduces the injury after intracerebral hemorrhage in the rat (Power et al., 2003). Hydroxymates, which are potent inhibitors to the MMPs through their binding to the active zinc site in the molecule, are effective in reducing edema in the rat model of intracerebral hemorrhage (Rosenberg and Navratil, 1997). A recent study showed that both direct injection of minocycline into the hemorrhage and giving it systemically was superior to systemic delivery (Xue et al., 2010). It will be interesting to see if these agents and others have an impact on the damage from the collagenase-induced cerebellar hemorrhage.

\section{Summary}

The model developed by Lekic and his colleagues provides a framework for a number of important studies on cerebellar hemorrhage. Using biochemical, histological and behavioral approaches they have mapped out the essential features of the model. Future directions will need to refine the behavioral studies to determine the sites in the cerebellum responsible for the behavioral effects. Are the biochemical changes produced by hemorrhage in cerebellar tissues similar to those in the basal ganglia? Will drugs that have a positive effect on supratentorial hemorrhage also work in the cerebellum? Do larger lesions produce brainstem compression with hydrocephalus, which would more closely model the acute illness in man? If so, can medical treatments reduce the secondary damage in such a model and prevent the mass effects that are now treated by surgical excision? This interesting report of a new model will stimulate interest in this important cause of stroke.

\section{References}

Broderick JP, Diringer MN, Hill MD, Brun NC, Mayer SA, Steiner T, Skolnick BE, Davis SM. Determinants of intracerebral hemorrhage growth: an exploratory analysis. Stroke. 2007; 38:10721075. [PubMed: 17290026]

Fisher CM, Picard EH, Polak A, Dalai P, Ojemann RG. Acute hypertensive cerebellar hemorrhage: Diagnosis and surgical treatment. J. Nerv. Ment. Disord. 1965; 140:38-57.

Gurney KJ, Estrada EY, Rosenberg GA. Blood-brain barrier disruption by stromelysin-1 facilitates neutrophil in.ltration in neuroinflammation. Neurobiol. Dis. 2006; 23:87-96. [PubMed: 16624562]

Hua Y, Keep RF, Gu Y, Xi G. Thrombin and brain recovery after intracerebral hemorrhage. Stroke. 2009; 40:S88-S89. [PubMed: 19064789]

Lekic T, Rolland W, Hartman H, Kamper J, Suzuki H, Tang J, Zhang JH. Characterization of the brain injury, neurobehavioral pro.les, and histopathology in a rat model of cerebellar hemorrhage. Exp. Neurol. 2011; 227:96-103. [PubMed: 20887722]

Maier CM, Hsieh L, Yu F, Bracci P, Chan PH. Matrix metalloproteinase-9 and myeloperoxidase expression: quantitative analysis by antigen immunohistochemistry in a model of transient focal cerebral ischemia. Stroke. 2004; 35:1169-1174. [PubMed: 15060315]

Montaner J, Alvarez-Sabin J, Molina CA, Angles A, Abilleira S, Arenillas J, Monasterio J. Matrix metalloproteinase expression is related to hemorrhagic transformation after cardioembolic stroke. Stroke. 2001; 32:2762-2767. [PubMed: 11739970]

Ott KH, Kase CS, Ojemann RG, Mohr JP. Cerebellar hemorrhage: diagnosis and treatment. A review of 56 cases. Arch. Neurol. 1974; 31:160-167. [PubMed: 4546748]

Power C, Henry S, Del Bigio MR, Larsen PH, Corbett D, Imai Y, Yong VW, Peeling J. Intracerebral hemorrhage induces macrophage activation and matrix metalloproteinases. Ann. Neurol. 2003; 53:731-742. [PubMed: 12783419] 
Powers WJ, Zazulia AR, Videen TO, Adams RE, Yundt KD, Aiyagari V, Grubb RL Jr, Diringer MN. Autoregulation of cerebral blood .ow surrounding acute (6 to 22 hours) intracerebral hemorrhage. Neurology. 2001; 57:18-24. [PubMed: 11445622]

Rosenberg GA, Navratil M. Metalloproteinase inhibition blocks edema in intracerebral hemorrhage in the rat. Neurology. 1997; 48:921-926. [PubMed: 9109878]

Rosenberg GA, Mun-Bryce S, Wesley M, Kornfeld M. Collagenase-induced intracerebral hemorrhage in rats. Stroke. 1990; 21:801-807. [PubMed: 2160142]

Rosenberg GA, Dencoff JE, McGuire PG, Liotta LA, Stetler-Stevenson WG. Injury-induced 92-kDa gelatinase and urokinase expression in rat brain. Lab. Invest. 1994; 71:417-422. [PubMed: 7933992]

Rosenberg GA, Grossetete M, Mun-Bryce S. Experimental models in intracerebral hemorrhage. Chapter 15. Handb. Clin. Neurol. 2008; 92:307-324. [PubMed: 18790281]

Strick PL, Dum RP, Fiez JA. Cerebellum and nonmotor function. Annu. Rev. Neurosci. 2009; 32:413434. [PubMed: 19555291]

Xi G, Keep RF, Hoff JT. Mechanisms of brain injury after intracerebral haemorrhage. Lancet Neurol. 2006; 5:53-63. [PubMed: 16361023]

Xue M, Mikliaeva EI, Casha S, Zygun D, Demchuk A, Yong VW. Improving outcomes of neuroprotection by minocycline: guides from cell culture and intracerebral hemorrhage in mice. AmJPathol. 2010; 176:1193-1202.

Yang Y, Estrada EY, Thompson JF, Liu W, Rosenberg GA. Matrix metalloproteinase-mediated disruption of tight junction proteins in cerebral vessels is reversed by synthetic matrix metalloproteinase inhibitor in focal ischemia in ratJCereb. Blood Flow Metab. 2007; 27:697-709.

Zulch, KJ.; Minckler, J. Hemorrhage, thrombosis, embolism, pathology of the nervous system. McGraw-Hill Book Corp; New York: 1971. p. 1499-1535. 